\title{
Docosatrienoic Acid
}

National Cancer Institute

\section{Source}

National Cancer Institute. Docosatrienoic Acid. NCI Thesaurus. Code C68351.

A polyunsaturated very long-chain fatty acid with a 22-carbon backbone and exactly 3 double bonds. Double bonds for docosatrienoic acid can be originating from either the (3rd, 6th and 9th), the (5th, 11th and 17th), the (7th, 13th and 19th) or the (9th, 12th and 15th) positions from the methyl end. 